\title{
Numerical Evaluation of the Crack Compliance Method (CCM) in Beams With and Without Prior History
}

\author{
G. Urriolagoitia-Sosa ${ }^{1 a}$, G. Urriolagoitia-Calderón ${ }^{2 b}$, \\ J. M. Sandoval-Pineda ${ }^{1 c}$, L. H. Hernández-Gómez ${ }^{2 d}$, \\ E. A. Merchán-Cruz ${ }^{1 \mathrm{e}}$, R. G. Rodríguez-Cañizo ${ }^{1 f}$
} and J. A. Beltrán-Fernández ${ }^{2 g}$

\author{
${ }^{1}$ INSTITUTO POLITECNICO NACIONAL \\ Sección de Estudios de Posgrado e Investigación (SEPI), Escuela Superior de Ingeniería Mecánica \\ y Eléctrica (ESIME). Unidad profesional, AZCAPOTZALCO, \\ Av. de las Granjas No. 682, Col. Sta. Catarina Azcapotzalco, C.P. 02550, México \\ a guiurri@hotmail.com, c jsandovalp@ipn.mx, e eamerchan@ipn.mx, d rgrodriguez@ipn.mx
}

${ }^{2}$ INSTITUTO POLITECNICO NACIONAL Sección de Estudios de Posgrado e Investigación (SEPI), Escuela Superior de Ingeniería Mecánica y Eléctrica (ESIME). Edificio 5. 2do Piso, Unidad Profesional Adolfo López Mateos "Zacatenco"

Col. Lindavista, C.P. 07738, México, D.F. México

b urrio332@hotmail.com, d luishector56@hotmail.com

Key words: Prior Straining History, Residual Stresses, Crack Compliance Method, Finite Element Method.

\begin{abstract}
This work assesses the Crack Compliance Method (CCM), which has been extensively used for the experimental evaluation of residual stresses, by the Finite Element Method (FEM) to validate its experimental applicability through numerical evaluation. The CCM is a very powerful method that is based on Fracture Mechanics theory, but its experimental application and set up has not been totally scientifically validated. In this paper, a numerical evaluation is presented on the basic applications of the CCM. The assessment of the CCM is performed on bending beams with and without prior straining history. To determine the best position and orientation of the strain gages, as well as the optimum number of readings, a number of numerical simulations where also performed for the correct performance of the experimental evaluation of the CCM. The prior straining history condition, in the analyzed components, is induced by an axial pulling before the beam is bent. Three levels of preloading are considered: low, medium and high (which are related to the yield strain of the simulated material); Isotropic and Kinematic hardening rules are also considered. After the residual stress field is induced by bending, a slot cutting is simulated and the strain relaxation produced is captured, which is used later in the CCM program for the quantification of the original residual stress field. The results obtained in this work, provide a quantitative demonstration of the effect of hardening strain on the distribution of the residual stress in beams. In the same manner, the theoretical formulation of the CCM has been evaluated validating the application of this method for the determination of residual stress fields in mechanical components.
\end{abstract}

\section{Introduction}

The Crack Compliance Method (CCM) is based on the cutting of a thin slot of increasing depth into a specimen and measuring the nearby distortion produced using strain gauges [1]. It is assumed that the stresses do not vary along the length of the slot and that linear-elastic stress relaxation occurs 
during the cutting process. In this way, the direct residual stresses normal to the slot and, in some cases, the shear residual stresses parallel to the specimen surface, can usually be determined throughout the cut depth.

The CCM is used worldwide and it has been applied to various geometries. The most common specimens examined are beams and cylinders [2-4], which can be either solid or hollow [5]. Other geometries that can be tested include rectangular bars and brackets welded to a plate [6$8]$.

The reported advantages of the CCM over other destructive measurement methods include; a simple and rapid experimental implementation, since only a single growing slot is required for a test; greater accuracy due to the reduced degree of machining, as repetitive processes can accumulate errors and even more new stress are introduced; and, a superior performance in measuring the localised residual stresses which result from any kind of manufacturing process [9].

The two main disadvantages of the method arise from the apparent difficulty in determining the appropriate crack compliance and the manner in which the slot is induced into a component. For the crack compliance, the solution obtained analytically can involve quite considerable mathematics; whilst those calculated using FEM analysis require a new model for each depth increment [10].

When a slot is induced into the specimen, there are several issues about the correct manner to support the specimen before the cut is performed and the optimum location to place the strain gauge. This work focuses in these two issues and will suggest some solutions. In this paper, the numerical evaluation of the CCM has been presented. Until now, the CCM it has been only applied and assessed experimentally. In the opinion of the authors, there is deficient information about the correct manner of performing the experimental procedure and to be convinced that the results obtained are the optimum ones. Perhaps, numerical analysis performed by FEM could give the answers referent of all these issues concerning the CCM, because applying a numerical evaluation will not introduce external factors that can affect the recalled strain data needed to perform it.

\section{The global polynomial residual stress field inverse method}

Consider a strip specimen of depth $t$ and unit thickness, (Figure 1) [11].

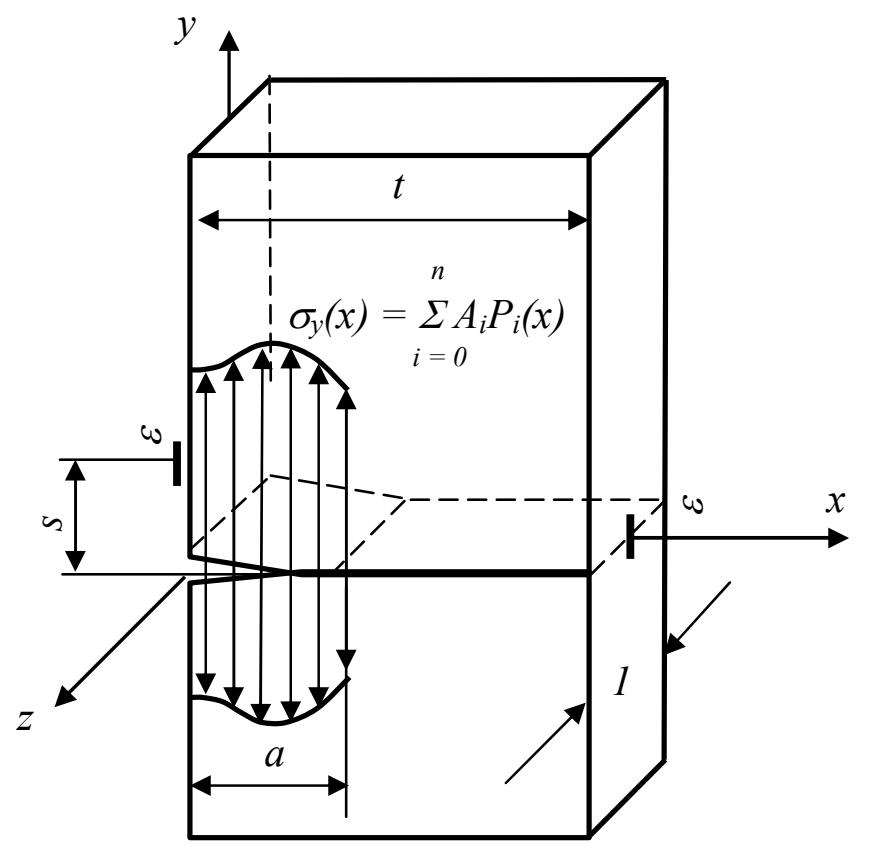

Figure 1.- Measurement of strains near the cut or on the back face [11] 
The surface traction $\sigma_{y}(x)$ is the unknown residual stress that has to be deduced from measurement of the strain $\varepsilon$ at some location (for example $x=0, y=s$ or at $x=t, y=0$ ). Furthermore, let the unknown residual stress distribution in the beam to be represented as an $n^{\text {th }}$ order polynomial series [11]:

$$
\sigma_{y}(x)=\sum_{i=0}^{n} A_{i} P_{i}(x)
$$

where $A_{i}$ are the coefficients that have to be obtained and $P_{i}$ are a power series, $x^{0}, x^{1}, x^{2}, \ldots . x^{n}$ etc., Legendre polynomials are also used. However, the crack compliance method includes a step which assumes that a stress distribution, $\sigma_{y}(x)=P_{i}(x)$, interacting with the crack is known. This known stress field is used to obtain the crack compliance function $C$ by using Castigliano's approach.

To illustrate the determination of the compliance functions, a strip of unit thickness and unit width in the $z$ direction with an edge crack of length $a$ (Figure 2), is considered. In order to obtain the horizontal displacement $u$ at $(l, s)$, a pair of virtual forces $F$ are introduced at that location in the horizontal direction. The forces may be located at either the top or bottom surface. The change in the strain energy due to the presence of the crack and the virtual force is given by [3]. (where $K$ are the stress intensity factors due to the surface tractions $K_{I}$ and the virtual force $K_{I F}$ ):

$$
U=\frac{1}{E^{\prime}} \int_{0}^{a}\left(K_{I}+K_{I F}\right)^{2} d a
$$

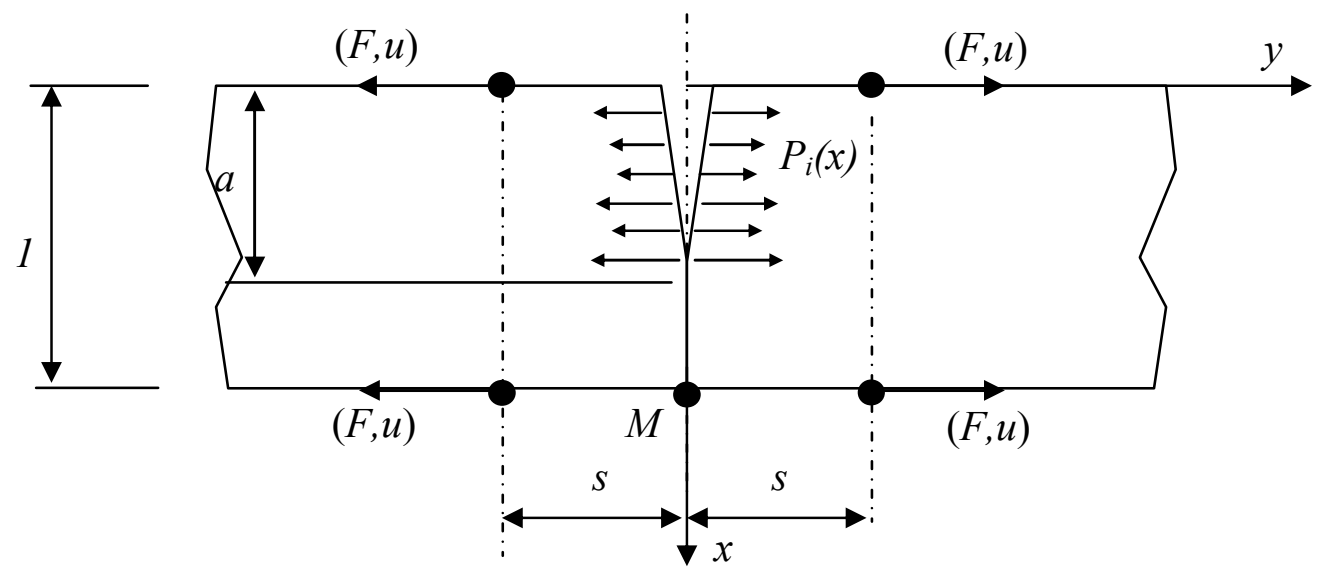

Figure 2.- Edge-Cracked strip subjected to surface loading and virtual force [3]

Applying Castigliano's theorem, the displacement $u(a, s)$ can be determined by taking a derivative of the strain energy with respect to the virtual force, as [11]:

$$
u(a, s)=\left.\frac{1}{2} \frac{\partial U}{\partial F}\right|_{F-0}=\left.\frac{1}{E^{\prime}} \int_{0}^{a} K_{I} \frac{\partial K_{I F}(a, s)}{\partial F} d a\right|_{F=0}
$$

Differentiating now with respect to the distance $s$ [11]:

$$
\varepsilon\left(a_{j}, s\right)=\frac{1}{E^{\prime}} \int_{0}^{a} K_{I}(a) \frac{\partial^{2} K_{I F}(a, s)}{\partial F \partial s} d a
$$

This strain $\varepsilon(a, s)$ due to the stress $P_{i}(x)$ is known as the compliance function $C_{i}(a, s)$ so that;

$$
C_{i}\left(a_{j}, s\right)=\frac{1}{E^{\prime}} \int_{0}^{a_{j}} K_{I}(a) \frac{\partial^{2} K_{I F}(a, s)}{\partial F \partial S} d a
$$

Due to the linearity of $K_{I F}$ with $F$, the second term under the integral in Equation 5 is the same as $Z(a)$ in $Z(a)=\frac{B}{F}\left(\left.\frac{\partial K_{I F}}{\partial S}\right|_{S=0}\right)$ with $B=1$, Therefore, it can be written as: 


$$
C_{i}\left(a_{j}, s\right)=\frac{1}{E^{\prime}} \int_{0}^{a_{j}} K_{I}(a) Z(a) d a
$$

By following the approach in Schindler et. al. [12] and Kang et. al. [13], for the case of a beam having the strain measurement point $M$ at the base, $K_{I}(a)$ and $Z(a)$ can be expressed as:

$$
\begin{gathered}
K_{I}(a)=\int_{0}^{a} h(x, a) \sigma_{y}(x) d x \\
Z(a)=4.283 \int_{0}^{a} h(x, a)(1-2 x) d x
\end{gathered}
$$

where $\sigma_{y}(x)=P_{i}(x)$ and $h(x, a)$ is known as the weight function [14]. The reference contains tabulated values of $h(x, a)$ for given values of $a$ and $x$. Therefore, $C_{i}\left(a_{j}, s\right)$ can be determine from Equation 8 by numerically integrating this expression. Once the $C_{i}(a, s)$ solutions have been obtained, the expected strain due to the stress components in Equation 1 can be determined as [11]:

$$
\varepsilon\left(a_{j}, s\right)=\sum_{i=0}^{n} A_{i} C_{i}\left(a_{j}, s\right)
$$

The unknown terms $A_{i}$ have to be determined so that the strains given by Equation 9 match those strains measured in the experiment. To minimise the average error over all data points for the $n^{\text {th }}$ order approximation, the method of least square is used to obtain the $A_{i}$ values. Therefore, the number of cutting increments, $m$, is often chosen to be greater than the order of the polynomials $P_{i}$ i.e. $m>n$. Typically $m=n+1$ is used [11]. This work used $n=8$ and $m=9$, the least square solution is obtained by minimising the square of the error relative to the unknown constant $A_{i}$, i.e. as in Equation 10 [11]:

This leads to

$$
\frac{\partial}{\partial A_{i}} \sum_{j=1}^{m}\left[\varepsilon(a, s)_{\text {actual }}-\sum_{k=0}^{n} A_{k} C_{k}\left(a_{j}, s\right)\right]^{2}=0 \quad i=0, \ldots ., n
$$

$$
[H]\{A\}=\{J\}
$$

where $[H]=[C]^{T}[C]$ and $\{J\}=[C]^{T}\left\{\varepsilon_{j}\right\}_{\text {actual }}$ [15]. Equation 11 gives a simple set of simultaneous linear equations. For the problems considered in this work, $[H]$ is a $8 x 8$ matrix. The numerical procedure was implemented in a FORTRAN program using the Compac Visual FORTRAN package. Therefore, equation 11 was solved using the LU Decompositor (LUDCMP) and Backsubstitution (LUBKSB) routines [16]. The actual residual stress distribution was then determined by using Equation 1.

\section{Numerical modelling of the beam}

The numerical evaluation of the CCM presented in this work is based on the residual stress induction applying pure bending. The beams are modelled in $2 \mathrm{D}$, in order to allow variations in stresses and strains through the depth to be determined. Quadratic order elements were used, in order to obtain smooth variations of stress and strain through the depth of the beams.

The beam was loaded (in all the cases) in a four-point bend configuration with the force located on two separate nodes, as illustrated in Figure 3a. The maximum value of the moment applied in each case was $9 \mathrm{Nm}$. Plastic deformation was obtained in all cases (without and with prior history).

Boundary conditions were applied at the extreme end nodes at the bottom (Figure $3 \mathrm{~b}$ ). Care was taken to produce small elements $(1 \mathrm{~mm}$ by $0.5 \mathrm{~mm})$ in the middle section of the beam, where the slot was simulated by deleting elements and the relaxed strain data was obtained for the numerical evaluation of the CCM. Ten divisions were used across the depth of the beam, to simulate 9 progressive cuts, leaving 1 element so that structural integrity would prevail.

Plane stress analysis was carried out and the loading was applied in a total of 100 sequentially increasing increments. The resulting strain in the $x$ direction, produced by the action of the bending loading was recorded for each loading increment. 


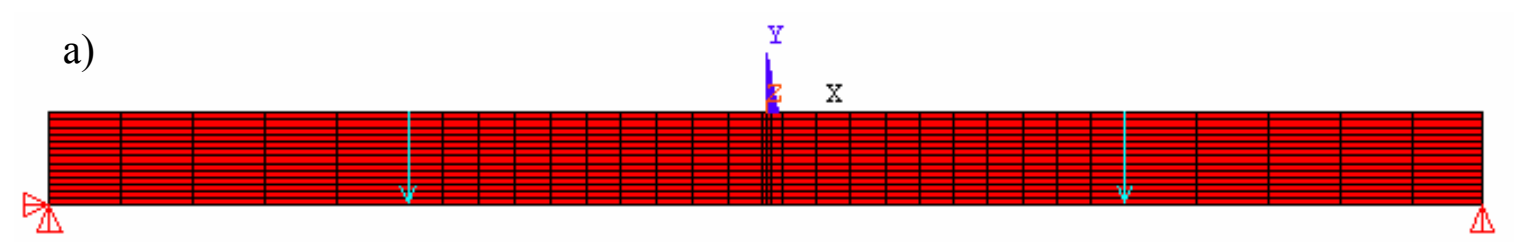

Dimensions in $\mathrm{mm}$

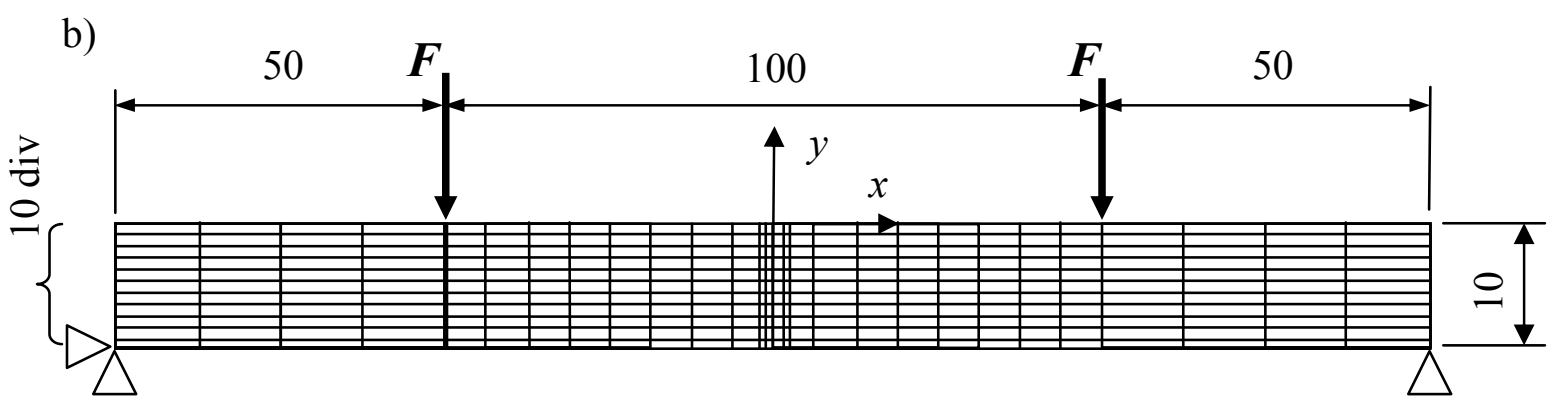

Figure 3.- Four-point bending beam model

a) FEA model b) schematic representation of the FEA model

The induction of prior history was done by pre-pulling the beam. For this purpose, three levels with respect to the yield strain were considered; light, medium and heavy. These correspond to a displacement in the direction $x$ of $0.8 \mathrm{~mm}, 2.0 \mathrm{~mm}$ and $2.8 \mathrm{~mm}$ respectively. The maximum total strains were 2, 5 and 7 times the value of the yield strain. A general material model consisting of a non-linear kinematic hardening and isotropic hardening components, as given in Equations 12 and 13 respectively [16], were used for the analyses.

$$
\begin{gathered}
d \alpha=C \frac{1}{\sigma_{o}}(\sigma-\alpha) d \bar{\varepsilon}^{p l}-\gamma \quad \alpha d \bar{\varepsilon}^{p l} \\
\sigma_{o}=\left.\sigma\right|_{o}+Q_{\infty}\left(1-e^{-\bar{\varepsilon}^{p l}} b\right)
\end{gathered}
$$

where ${ }^{-p l}$ is the equivalent plastic strain, $\alpha$ is the back-stress, $C$ is the initial kinematic hardening modulus, $\gamma$ determines the rate at which kinematic modulus decreases with plastic deformation, $\sigma_{o}$ is the current yield stress, $\left.\sigma\right|_{o}$ is the initial yield stress, $Q_{\infty}$ is the maximum change in the size of the yield surface and $b$ defines the rate at which the size of the yield surface changes as plastic straining develops. Equation 12 describes the translation of the yield surface in the stress space due to the back-stress, $\alpha$, while Equation 13 describes the change of the equivalent stress defining the size of the yield surface, $\sigma_{o}$, as a function of plastic deformation.

\section{Numerical evaluation of the CCM}

In this section the numerical evaluation of the CCM is developed. For all cases (with and without prior history) the same model of Figure 4 was considered.

Initially, CCM was simulated in a beam without a prior history. On the other hand, for the cases where prior history was considered, a homogenous pre-strain of the beam was applied before conducting the operation of bending. Moreover, in the considered cases, different hardening rules were applied, such as the isotropic hardening rule and the kinematics hardening rule, with which the mechanical behavior of the component is determined. The applied load and the boundary conditions are constant in each case of study. The mechanical properties established as input data are: $E=210$ $G P a, \sigma_{y}=420 \mathrm{MPa}$ and $v=0.29$. 


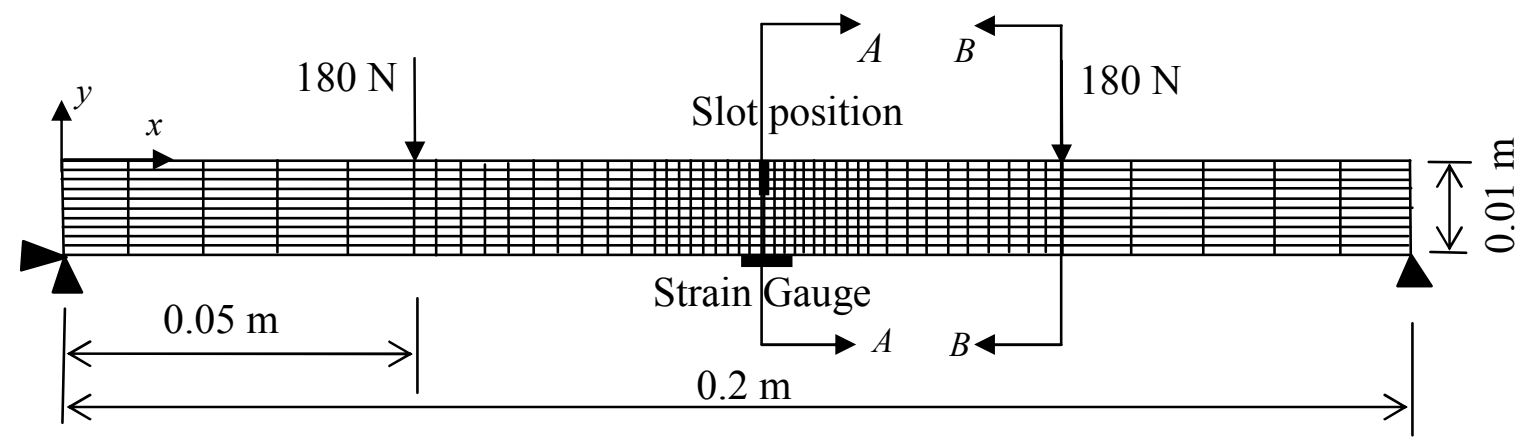

Figure 4. - Schematic representation of the model applied in the Finite Element Method

\section{Determination of the ideal position of the strain gauge in the beam}

For the experimental development of the CCM, it is important to find the ideal position of the strain gauge, because the accuracy of the measurement directly depends on this position. Therefore, the reading point in different positions with respect to the crack was simulated. Ten points at $1 \mathrm{~mm}$ increments were taken into account. The first one is at the crack plane (Figure 5).

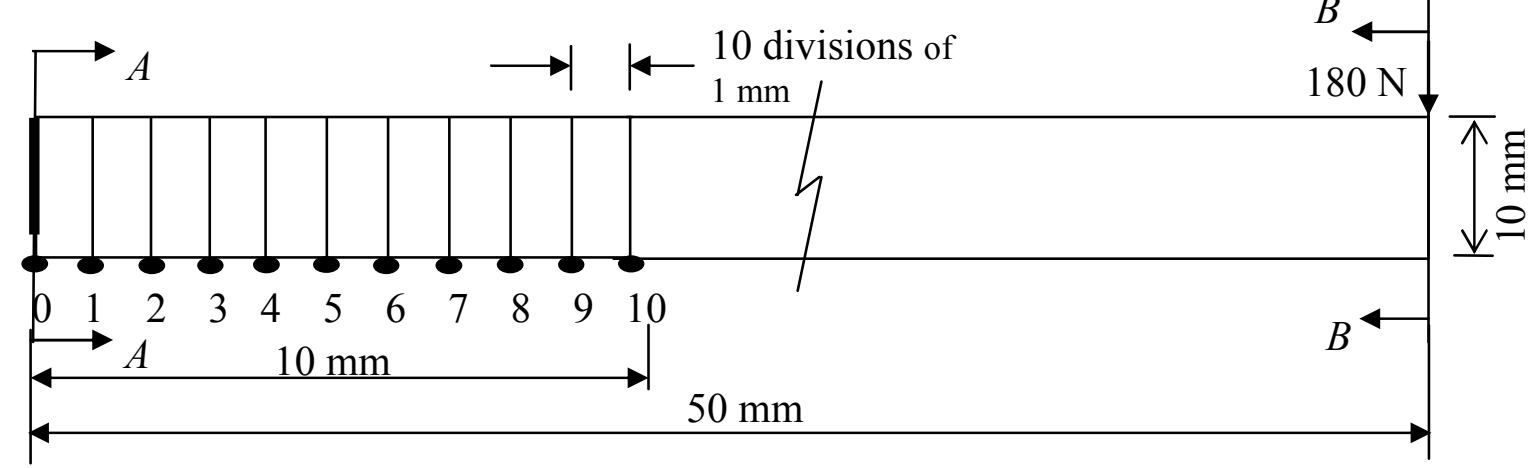

Figure 5.- Schematic localization of the reading points

The numerical results of the stress relaxation induced by the cut are shown in Figure 6 . This analysis was performed in a beam without prior history and under the isotropic hardening rule. Figure 7 shows the residual stress field obtained from the readings of Figure 6 . The residual stress fields obtained by the CCM are compared against those derived from the application of the FEM.

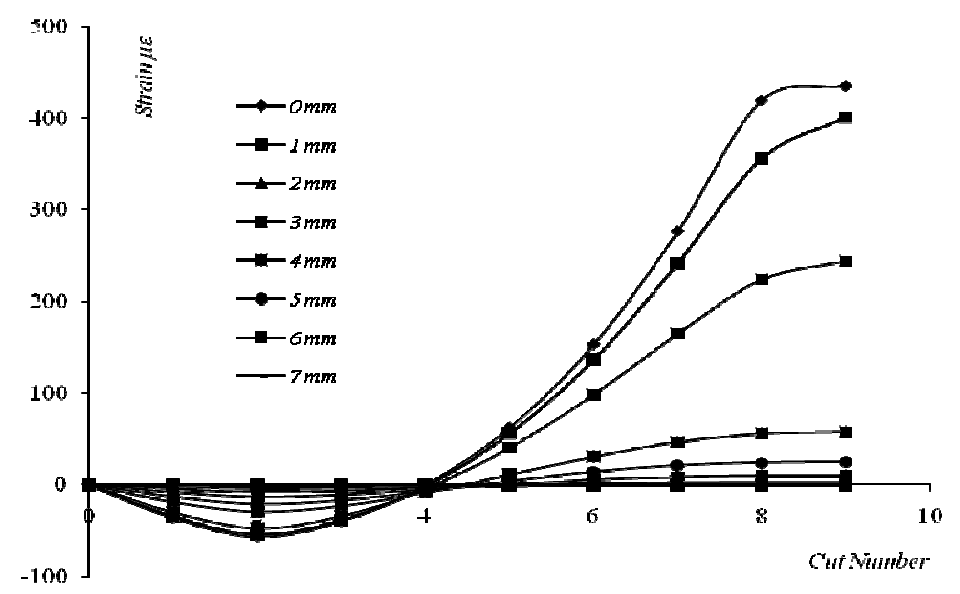

Figure 6. - Strain relaxation obtained at different positions along the beam 
It is important to indicate that the both numerical evaluations are close. It can also be concluded, that the optimum location of the gauge it is in the same plane of the crack and that it is possible to obtain the residual stress profile by using only one relaxed strain reading.

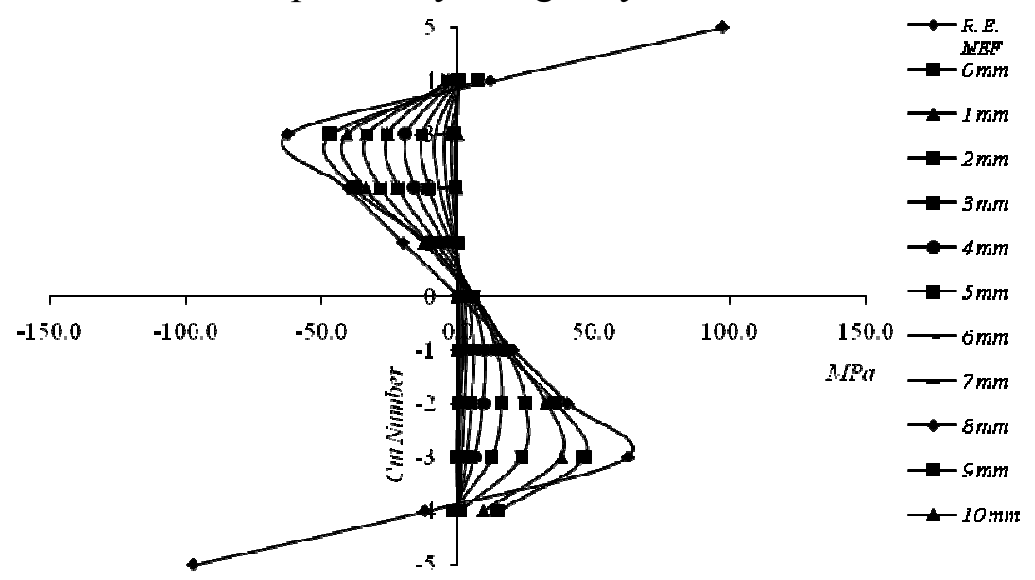

Figure 7. - Residual stress fields at different locations on the beam

\section{Numerical evaluation of the CCM under Isotropic hardening rule}

Figures 8 show the curves that represent the residual stress fields applying the CCM. These curves are superposed against the curves obtained by the FEM considering both prior history at different levels and no prior history. For all of these cases, the analysis was performed under the isotropic hardening rule.
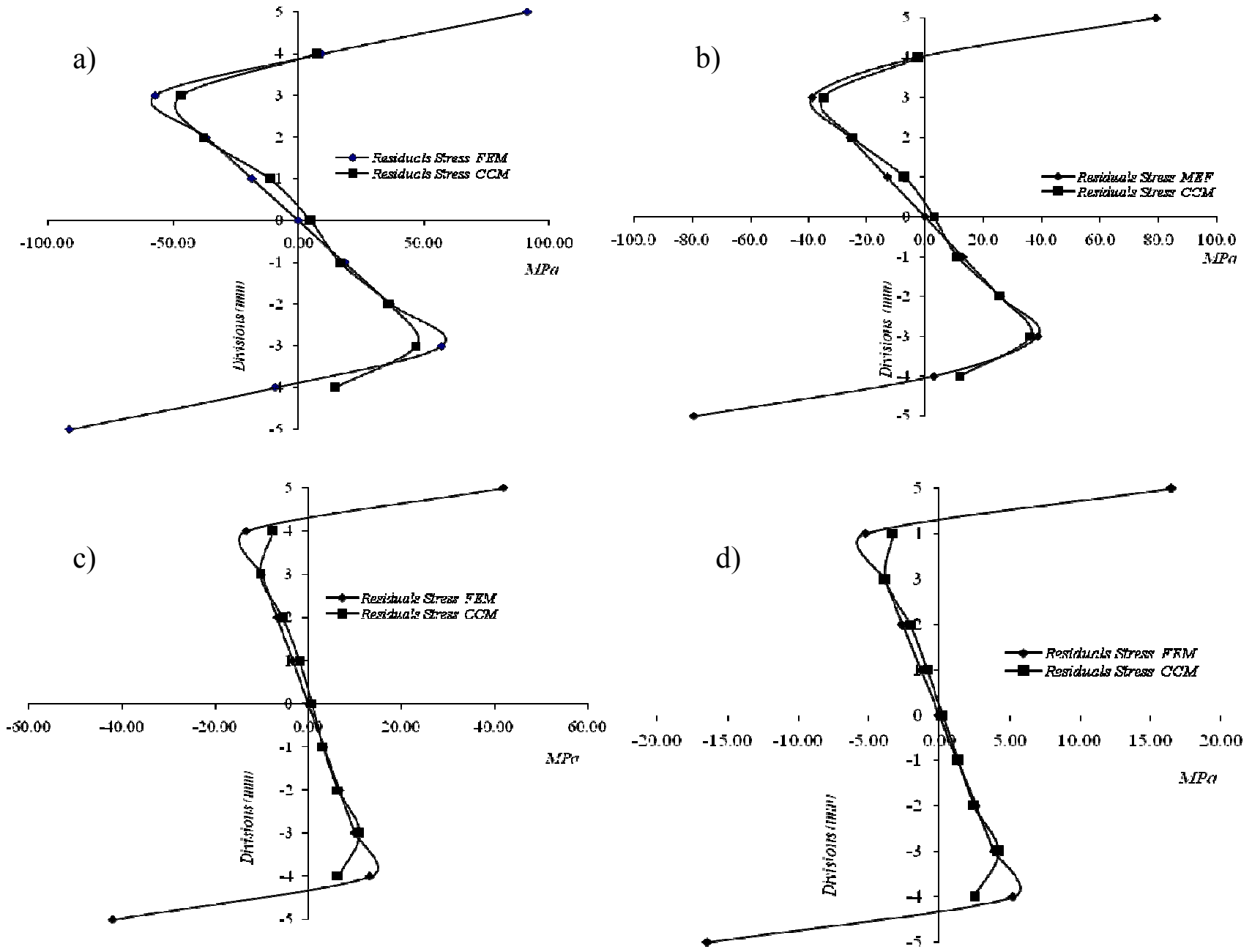

Figure 8.- Residual stress fields, isotropic hardening rule. a) Without prior history.

b) Prior history of $2 \varepsilon_{y}$. c) Prior history of $5 \varepsilon_{y}$. d) Prior history of $7 \varepsilon_{y}$. 


\section{Numerical evaluation of the CCM under Kinematic hardening rule}

In Figures 9 there are shown the residual stress results obtained from the measurement of the relaxation of the strain by introducing a cut and applying the CCM. They are compared with the numerical results, which are obtained with the kinematic hardening rule (without and with prior history), also considering strain hardening and Bauschinger effect as well [1].
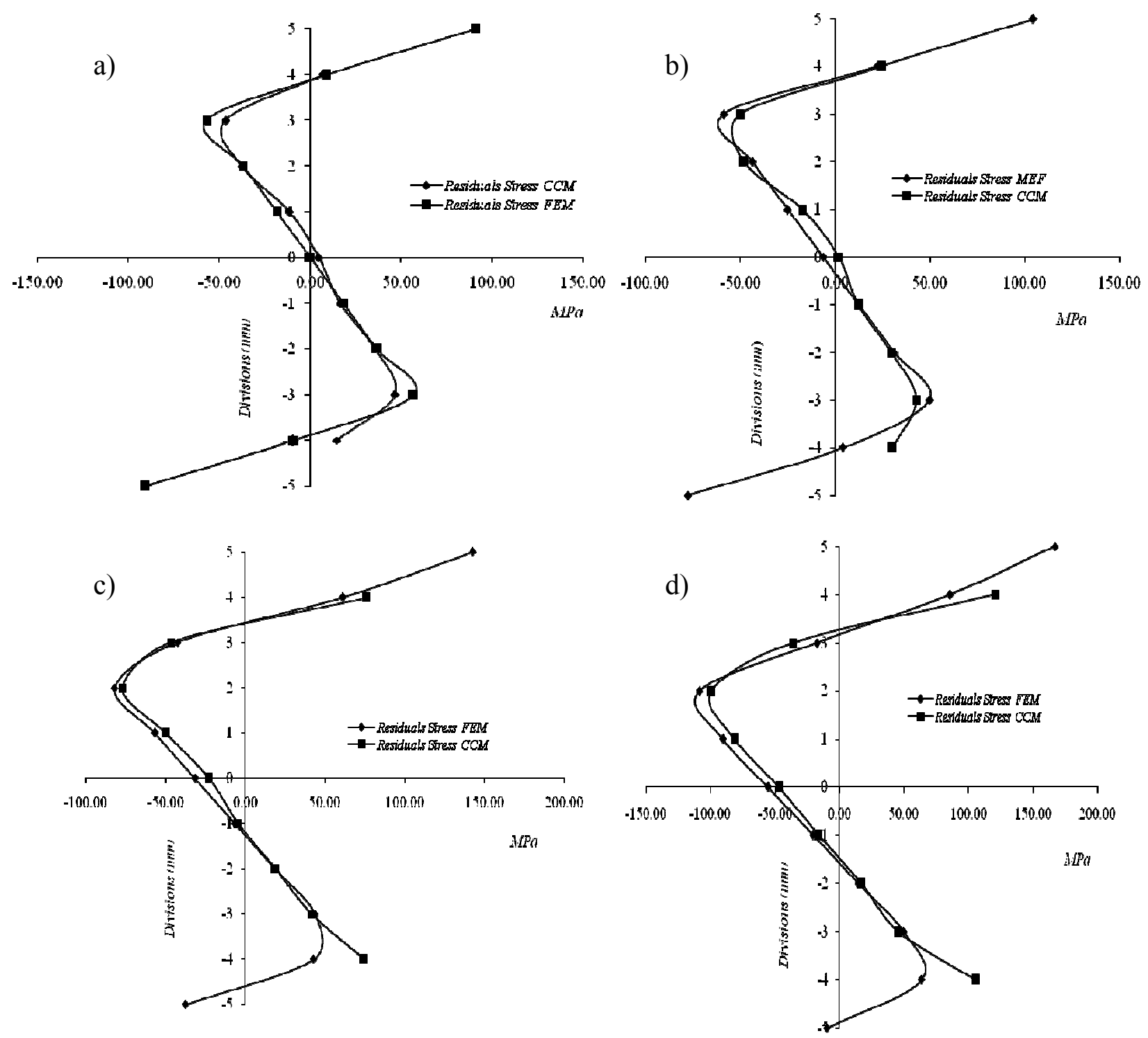

Figure 9.- Residual stress fields, kinematic hardening rule. a) Without prior history. b) Prior history of $2 \varepsilon_{\mathrm{y}}$. c) Prior history of $5 \varepsilon_{\mathrm{y}}$. d) Prior history of $7 \varepsilon_{\mathrm{y}}$.

\section{Discussions and conclusions}

In this paper, the numerical evaluation of the CCM has been presented. Until now, the CCM it has been only applied and assessed experimentally. In that sense, in the opinion of the authors, there is deficient information about the correct manner of performing the experimental procedure and to be convinced that the results obtained are the optimum ones. It is considered that, FEM could be the best option for the evaluation of the CCM, because there are not external factors that can affect the recalled strain data needed to perform it. Also, since the FEM simulation of the induction of the crack will not incorporate more stress into the component, due to the cutting process. 
On the other hand, Prime [17] and Nowell, et al, [6] have experimentally practice the introduction of the slot into the component by clamping one side of the specimen. But FEM results presented from this work, suggest that the experimental procedure on the beam or strip should be better completed if the component is free to relax (as much as possible). Urriolagoitia-Sosa [18] has developed a new and simple supporting system. This consists of two semi-cylindrical pieces upon which the beams analysed were placed. The pieces were simply placed on a smooth flat surface in the EDM cutting machine. The centres of the pieces were located at quarter distances from the two ends of a beam should be fully parted in two halves. Each one is capable of being supported by a semi cylindrical piece. The cylindrical shape of the pieces also allow them to rock as the beam is being cut and the internal residual stress relieved causes movement.

The numerical evaluation of the CCM will contribute in the selection of optimal location for the strain gauges and that it is possible to use only one strain reading to calculate the entire residual stress field. This will reduce the cost and time to develop and perform the experiment.

This evaluation was made by applying different hardening rules and different levels from prior history, to a rectangular beam under four points bending, onto which later fields of residual stress were induced.

The paper has presented the numerical evaluation and application of the CCM developed for the study of residual stresses in beams that were pre-strained before residual stresses were mechanically induced through bending. The results obtained from the CCM agree closely with those obtained using the FEM of superposition of loading and unloading stresses. As expected, residual stresses developed in beams with prior straining, shows asymmetry in magnitude of the stresses on the tensile and compressive sides.

\section{Acknowledgment}

The authors gratefully acknowledge the financial support from the Mexican government by de Consejo Nacional de Ciencia y Tecnología and the Instituto Politécnico Nacional.

\section{References}

[1] Urriolagoitia-Sosa, G, Analysis of prior strain history effect on mechanical properties and residual stress in beams, Thesis Ph D, Oxford Brooks University, pp 129-142. (2005)

[2] Cheng, W. and Finnie, I., A method for measurement of axisymmetric residual stresses in circumferentially welded thin-walled cylinders, $J$ of Enginnering Materials and Technology, Vol. 107, pp 181-185. (1985)

[3] Cheng. W., Prime, M., B. and Finnie, I., Measurements of residual stresses through the thickness of a strip using the crack compliance method, Residual Stress III - Science and Technology Proceedings $3^{\text {rd }}$ International Conference of Residual Stress, Vol. 2, pp 1127-1132. (1997)

[4] Schindler, H. J. and Landolt, R., Experimental determination of residual stress and the resulting stress intensity factors in rectangular plates, $4^{\text {th }}$. European conference on residual stresses, Cluny, France, pp 509-517. (1997)

[5] Cheng, W. and Finnie, I., Measurement of residual hoop stress in cylinders using the compliance method, Journal of engineering materials and technology, Vol.108, pp 87-92, (1986)

[6] Nowell, D., Tochilin, S. and Hills, D. A., Measurement of residual stress in beams and plates using the crack compliance technique, Journal Strain Analysis, Vol. 35, No. 4, pp 277-285, (2000)

[7] Schindler, J. H., Residual stress measurement in cracked components: Capabilities and limitations of the cut compliance method, Materials science forum, Vols 347-349, pp 150-155. (2000) 
[8] Urriolagoitia-Sosa, G., Durodola, J. F. and Fellows, N. A., Determination of residual stress in beams under Bauschinger effect using surface strain measurements, Strain, Vol. 39, No. 4, Nov, pp 177-185, (2003)

[9] Prime, M., B., Residual stress measurement by successive extension of a slot: The crack compliance method, Applied mechanics reviews, Vol. 52, No. 2, pp 75-96. (1999)

[10] Prime, M. B., Rangaswamy, P. and Bourke, M. A. M., Measuring spatial variation of residual stresses in a MMC using crack compliance, $7^{\text {th }}$ International Conference on Composites Engineering, pp. 711-712. (2000)

[11] Cheng, W. and Finnie. I., An overview of the crack compliance method for residual stress measurement, Proc. $4^{\text {th }}$ Int. Conf. Residual Stress, Baltimore, Maryland, Society for Experimental Mechanics, pp 449-458. (1994)

[12] Schindler, H. J., Cheng, W. and Finnie, I., Experimental determination of stress intensity factors due to residual stresses, Journal of experimental mechanics, Vol. 37, No. 3, pp 272-277. (1997)

[13] Kang, K. J., Song, J. H. and Earmme, Y. Y., A method for the measurement of residual stresses using a fracture mechanics approach, Journal of strain analysis, Vol. 24, 23-30. (1989)

[14] Wu, X. R. and Carlsson, A. J., Weight function and stress intensity factor solutions. Pergamon Press, Oxford, England, (1991)

[15] Press, W. H., Flannery, B. P., Teukolsky, S. A. and Vetterling, W. T., Numerical recipes, Cambridge University Press. (1987)

[16] ABAQUS, Version 6.3, Standard user's manual, Vol. II. (2002)

[17] Prime, M. B., Experimental procedure for crack compliance (slitting) measurements of residual stress, Los Alamos National Laboratory Report, LA-UR-03-8629, pp 1-6. (2003)

[18] Urriolagoitia-Sosa, G., Durodola, J. F. and Fellows, N. A., Effect of strain hardening on the residual stress distribution in beams measured using the crack compliance method, J. Strain Analysis for Engineering Design, Vol. 42, No. 2, pp 115-121, (2007) 